\title{
Spatio-Temporal Motility MRI (STMM) analysis of the stomach and colon
}

Alex Menys ${ }^{1 *}$, Caroline Hoad ${ }^{2,3^{* *}}$, Robin Spiller ${ }^{3}$, Mark Scott $^{4}$, David Atkinson ${ }^{1}$, Luca Marciani $^{3}$ \& Stuart Taylor ${ }^{1}$

1. Centre for Medical Imaging, UCL, $3^{\text {rd }}$ Floor Charles Bell House, London

2. Sir Peter Mansfield Imaging Centre, School of Physics and Astronomy, University of Nottingham, Nottingham UK

3. Nottingham Digestive Diseases Centre and National Institute for Health Research (NIHR) Nottingham Biomedical Research Centre, Nottingham University Hospitals NHS Trust and University of Nottingham, UK

4. Wingate Centre for Neurogastroenterology, Blizard Institute, QMUL, London

* Corresponding Author

** Co-first

Alex Menys is the CEO of Motilent Limited

\section{Corresponding Author Details}

Dr Alex Menys

alex.menys.09@ucl.ac.uk

2nd Floor Charles Bell House

43-45 Foley Street,

London, W1W 7TS

Direct Contact: 02076798156

Fax: NA 


\section{Abstract}

Background:

MRI is increasingly used to objectively assess gastrointestinal motility. However, motility metrics often do no not offer insights into the nature of contractile action. This study introduces a systematic method of making spatiotemporal measurements of contractions, based on changes in bowel lumen diameter.

Methods:

Two heterogeneous cohorts of subjects were selected displaying gastric $(n=15)$ and colonic motility $(n=20)$ on which to test the Spatio-Temporal Motility MRI (STMM) technique. STMM involved delineating the bowel lumen along with inner and outer bowel wall along a section of the gastrointestinal tract. A series of diameter measurements were made automatically across the central axis of the lumen. Measurements were automatically propagated through the time series using a previously validated algorithm. Contractions were quantitatively summarised with two methods measuring 1) Normalised Contraction Plot (NCP) and 2) Combined Velocity Distance (CVD) both of which can be visualised as spatiotemporal motility maps. Both metrics were correlated against subjective visual scoring systems.

Key Results:

Good correlation was seen between reader scores and both motility metrics (NCP, $\mathrm{R}=0.85, \mathrm{P}<0.001, \mathrm{CVD}, \mathrm{R}=0.93, \mathrm{R}<0.001$ ) in the gastric data. Good correlation was also seen between the reader scores and the two metrics in the colonic data (NCP, $\mathrm{R}=0.82, \mathrm{P}<0.001, \mathrm{CVD}, \mathrm{R}=0.78, \mathrm{R}<0.001$ ).

Conclusions \& Inferences:

STMM analysis of the stomach and colon correlates well with reader scores in a range of datasets and provides both a quantitative and qualitative means of assessing contractile activity in the gastrointestinal tract

\section{Key points:}

1. Quantified assessment of gastrointestinal motility using MRI is increasingly common. This method is a powerful means of observing if the bowel is moving but to date does not tell us about more complex aspects of motility like coordination. Manometry remains the gold-standard.

2. This paper applies a spatiotemporal mapping technique to MRI data for the first time enabling systematic measurements of gastric and colonic lumen diameter presenting the data as a spatiotemporal map that might be evaluated subjectively or quantitatively.

3. Good correspondence between the MRI derived spatiotemporal maps and consensus visual assessment was seen and represents the first step towards improved phenotyping of GI tract motility in health and disease. 


\section{Introduction}

Gastrointestinal motility is complex and highly variable both within and between individuals in both health and disease. One of the key challenges faced by the research and clinical communities is the relative paucity of investigative tools(1). In both the upper and lower GI tract Manometry is still considered the gold standard for detailed analysis of motility, based on the assessment of intraluminal pressure change, but is invasive and burdensome from a time and cost perspective.

MRI is increasingly advocated as a safe and non-invasive tool to evaluate GI motility, and a growing literature reports that altered motility can be assessed using MRI in various disease states(2-7). However, despite a number of technical advancements enabling reproducible quantification of the data, motility analysis remains crude, essentially classification into hyper and hypo motile contractile states(8-10). Such analysis may be sufficient in some disease conditions, notably Crohn's disease, where hypomotility is a biomarker of inflammatory burden $(2,11,12)$. However, it is likely that a simple, 'high-low' measure of motility is insufficient to appropriately capture the inherent complexities of dysmotility in vivo in conditions like constipation(13). Indeed, aberrant coordination of contractile activity is often a key feature of dysmotility recorded by high resolution manometry(14). Ideally MRI tools would be able to capture the specifics of contractile information along the GI tract as spatio-temporal information similar to what we see with manometry except measuring luminal distances instead of pressure.

This proof of concept study provides a methodological overview of a novel semiautomated technique for capturing and quantifying luminal motility at high spatio-temporal resolution using dynamic 'cine' MR images. Initial validation is provided by applying the technique in the stomach and colon and two new summary motility metrics are proposed.

\section{Materials and Methods}

\section{Subject selection \& MRI data acquisition}

After appropriate ethical permissions, historical datasets were selected from the databases of two donor institutions.

Gastric datasets were captured by University College London Hospitals' Ethical Approval (Hampstead REC 10/H0720/91) and the colonic datasets were covered by University of Nottingham's Ethical Approval (EudraCT Number 2010-02187985, National Research Ethics Service (approval 10/H0906/50), the NHS Trust R\&D (approval 10GA018)).

Gastric motility: The anonymised MRI datasets of 50 Crohn's disease patients undergoing MR enterography as part of their usual clinical care were reviewed by 
the study coordinator. The datasets all contained coronal cine motility sequences focused on the small bowel, which are performed routinely by the donor institution. Specifically, each patient had consumed 2\% mannitol solution before being scanned in the prone position on either a 1.5T Siemens Avanto or 3T Philips Achieva scanner. On both scanners motility was captured using a balanced gradient echo sequence with a temporal resolution of $1 \mathrm{~s}$ over the course of a $20 \mathrm{~s}$ breath hold (full sequences provided in Appendix 1).

The study coordinator identified datasets where the whole gastric volume had also been captured by the dynamic motility series. Overall 27 datasets were excluded as they did not include the stomach. A further sub-set were rejected due to artefact (e.g. balanced gradient echo sequence banding,) $(n=5)$ or evidence of substantial respiratory motion $(n=7)$, leaving a total of 15 subjects ( 9 female median age 29 (range 20 to 46)) for inclusion.

Colonic motility data: The datasets of 20 healthy subjects undergoing MRI before and after ingestion of either $1 \mathrm{~L}(\mathrm{~N}=10)$ or $2 \mathrm{~L}(\mathrm{~N}=10)$ of polyethylene glycol (PEG) (Macrogol 3550) electrolyte solution (Moviprep ${ }^{\circledR}$; Norgine Pharmaceuticals Ltd, Harefield, UK) were reviewed by $\mathrm{CH}$. This cohort has been previously described in Hoad et al(15) and was selected given the known stimulatory effect of polyethylene glycol on the colon, which in its resting state is relatively inert if observed over a short duration. Moviprep ${ }^{\circledR}$ also provides good contrast to the colon lumen and as well as distension.

Imaging was carried out supine in a $1.5 \mathrm{~T}$ Philips Achieva scanner. Motility was captured in the ascending colon using a single sagittal slice balanced turbo field echo sequence, positioned centrally within the lumen of the right colon. Motility was captured during free-breathing for 120 seconds at a temporal resolution of 1 image per second.

Datasets were reviewed and those with good quality images scans through the centre of the ascending colon (AC) and visible colonic wall motion were selected

A total of 20 datasets (10 subjects scanned twice, pre and post polyethylene glycol ingestion) were selected (6 Female, median age 22 years, range 19 to 50).

\section{Data preparation and pre-processing}

All data was processed with the GIQuant ${ }^{\circledR}$ (Motilent, London, UK) motility registration technique by the study coordinator. In brief, image registration produces a series of deformation fields that can be used to propagate a region of interest through a time series of ' $n$ ' images in an automated fashion. To date, these deformation fields have been summarised to provide a surrogate of motility (e.g. the Standard Deviation of the Jacobian score $)(8,16)$.

In the current report, the deformation fields enabled the secondary use of the spatio-temporal mapping technique Spatio-Temporal Motility MRI (STMM) described below. As the gastric motility data was processed in breath-hold 
respiratory correction was not required in these data. Conversely, the colonic data was collected during free-breathing and respiratory correction was used here to increase the fidelity of the bowel wall motion correction. In summary, the technique previously referred to in the literature as 'Dual Registration of Abdominal Motion(17)' was applied which has previously been implemented in several studies assessing colonic motility data $(7,15)$.

\section{The Spatio-Temporal Motility MRI (STMM) technique}

Spatio-Temporal Motility MRI (STMM) describes a method to make automated, systematic measurements of the cross-sectional diameter of the GI tract. In this study the user

1. Manually delineates the midline (M) of the bowel on a single time frame image;

2. Manually delineates the bowel wall ( $\mathrm{L}_{1}$ and $\left.\mathrm{L}_{2}\right)$ either side of the bowel;

3. STMM automatically generates a series of nodes $(\mathrm{N})$ at pre-determined intervals along the midline (here a spacing of 2 pixels was used);

4. STMM generates a 1D line diameter measurement (D) [D $1-\mathrm{N}$, perpendicular to the midline at each $\mathrm{N}$ position. $\mathrm{D}$ is terminated when it intersects with the two outer bowel wall lines (Figure 1a);

The deformation fields generated by GIQuant ${ }^{\circledR}$ during the registration are thereafter used to propagate the coordinates of midline, lumen and nodes through the dynamic time series in a fully automated fashion. As the bowel wall moves (i.e. relaxes or narrows), the length of the perpendicular line at that position will change at that time point. The length of each perpendicular line is recorded at each time point throughout the series.

Over the time series a 2D spatio-temporal matrix is constructed to quantify changes in bowel diameter (X axis representing time and $\mathrm{Y}$ representing the position of the diameter measurement along the region of bowel under investigation. These data can be interpreted in a way similar to manometry in the sense that we get measures at regular intervals long the long axis of the tract. 


\section{Spatio-temporal plot analysis}

We propose two different methods of generating and quantifying the spatiotemporal plots.

Metric 1: Normalised Contraction Plot (NCP).

Purpose: To visualise and quantify luminal change in diameter over time.

Rationale: This metric captures the reduction in luminal diameter at a given node position for each time point. The mean diameter of the GI tract lumen is variable at differing anatomical locations. (e.g. the diameter of the stomach near the pylorus is less than at the fundus). Therefore, the data needs to be normalised such that relatively large contractions in narrower regions of bowel are not obfuscated by relatively small contractions in part of the bowel with a larger calibre. To normalise the data, a polynomial line of best fit was made through the time points at each node position. The error between the fit and the actual data was calculated and plotted (ERR). To account for underlying noise in the plots, only contractions over an empirically selected 5\% change in mean negative diameter were quantified informed by previous investigations in contractility assessment with MRI(18).

Summary statistic: The total negative change in diameter over the pre-set threshold is calculated for each node position (x-axis of plot). The average of each node's AUC is then averaged for all of the node positions to produce a single, unitless, summary statistic (Figure $2 \mathrm{~B}+\mathrm{E}$ and Figure $4 \mathrm{~B}+\mathrm{E}$ ). The higher the value the greater the amount of bowel wall motion in the series.

Metric 2: Combined Velocity \& Distance (CVD) Plot

Purpose: To visualise and quantify expansion and contraction of the bowel i.e. positive and negative luminal diameter changes of the bowel wall over time.

Rationale: This metric is particularly suited to the colon as positive and negative luminal diameter changes often occur. Thus the position of the wall following a contraction may not be the same as the start. The speed of the wall motion is calculated from a smoothed (kernel 7 in time domain) plot of the change in each line length over time. This parameter is combined with the absolute normalised contraction plot data defined for metric 1 using the equation below which generates only positive value data:

\section{Combined data score $=$ speed $^{*} \mid$ ERR $\mid$}

To remove the underlying noise in the plots (generated from the DRAM registration correction of the free breathing) and the summary statistic, a minimum threshold level of 0.375 of the combined data score is defined. This was set empirically using datasets which showed no visible wall movement and only small residual motion from breathing following DRAM registration. Only data above this threshold is shown on the plot and used in the summary statistic. 
Summary statistic. The total AUC under the plot is calculated for all data above the minimum threshold and the value is normalised to a typical bowel length of 20 lines defined for the fixed node spacing of 2 pixels used here as a compromise between spatial resolution and computation time. The higher the value the greater the amount of bowel wall motion in the series.

\section{Statistics}

All data were checked for normality using Shapiro-Wilk and appropriate correlative statistics used to assess the agreement between motility metrics all in R (Version 3.4.4, Vienna, Austria).

Gastric motility was visually assessed through consensus by experienced readers (AM and $\mathrm{CH}$ ) on a score of 1 (low motility) to 3 (high motility) with the two readers results averaged to produce a reference standard. The level of agreement was assessed with Intra-Class Correlation. Gastric motility derived using the two methods were correlated against the reader reference standard and against each other.

Colonic motility was visually assessed using a semi-quantitative visual colonic motility grading system as reported by Marciani et al(19). As with the gastric study, both motility metrics were correlated against the visual score and against each other.

Correlation statistics were used with values $<0.05$ considered as statistically significant. 


\section{Results}

\section{Gastric motility}

A visual overview of the gastric study is presented in figure 2. Comparison of the two readers scores produced an intra-class correlation coefficient of $0.8(\mathrm{P}<$ 0.001).

The averaged reader scores produced a median motility score of 2 (range 1 to 3). The median Normalised Contraction Plot (NCP) and Combined VelocityDeformation (CVD) score were 3 (range 0.2 to 16) and 67 (range 2.5 and 411 ) respectively.

Correlation of the visual score against the NCP was significant $\mathrm{R}=0.85(\mathrm{P}$ $<0.01$ ).The correlation between visual score and CVD map was also significant $R$ $=0.93(\mathrm{P}<0.001)$ (Figure 3).

Correlation of the two spatio-temporal motility metrics with each other produced a significant correlation of $R=0.944(P=0.001)$. 
Colonic motility

A visual overview of the colonic study is presented in Figure 4. The median visual score was 16 (range 0 to 363), the median NCP score was 22 (range 0 to 60) and median CVD score was 22 (range 0 to 572).

The visual score and NCP produced a positive correlation of $\mathrm{R}=0.82(\mathrm{P}<0.001)$. The visual score against CVD score also produced a significant correlation of $\mathrm{R}=$ $0.78(\mathrm{P}<0.001)$ (Figure 5).

Correlation of the two spatio-temporal motility metrics with each other produced a sginificant correlation of $\mathrm{R}=0.89, \mathrm{P}<0.001$. 


\section{Discussion}

The purpose of this study was to propose and provide preliminary validation of a novel MRI spatio-temporal mapping technique to objectively capture contractile activity in the gastrointestinal tract. We focused on the stomach and ascending colon where the lumen could be clearly visualised and demonstrated excellent agreement against the reference-standard observations of an experienced reader.

High-resolution manometry (HRM) remains the current reference standard for capturing gastrointestinal motility(20). Clinical uptake has been highest in the oesophagus and anorectum, both easily accessible and where clinically relevant physiological data can be extracted from relatively short sections of the GI tract(20). Conversely, the use of manometry in the small bowel and colon remains less widely used due to its invasiveness and the cost associated with accessing these deeper regions of the GI tract(21). Beyond the practical challenges of intubation, the intrinsic complexity of these large organs themselves remains a fundamental barrier for our understanding motility in both health and disease. Even in the stomach, the anatomical variability means catheter and pressure sensor placement can be inconsistent making interpretation challenging.

The underlying premise of manometry however remains appealing; to visualise and measure pressure-change as a surrogate of gastrointestinal activity. We know that coordination of contractile events in the bowel is important for its proper function and disruptions may underpin a range of gastrointestinal complaints. However, our understanding is limited by the inability to easily observe or measure this activity.

MRI may address at least some of the limitations of manometry. It is non-invasive, increasingly available and the growing array of post-processing techniques is facilitating easy and automated quantitation of gastrointestinal motility $(8,9,17,22)$. Although a number of challenges are yet to be overcome, not least the most effective patient preparation and data acquisition protocol practicable in clinical practice, the ability to quantify global or regional motility from one dataset is a significant advantage(16). MRI motility assessment has already enjoyed relative success in Crohn's disease (as biomarker of inflammatory activity), chronic constipation, and functional dyspepsia(2,7,11,12,23). To date however most work has explored if the bowel can contract in a largely binary manner. The next logical step is to extend the technique to better characterise the way the bowel contracts in terms of coordination.

Our method is inspired by manometry, although clearly is not capable of measuring pressure (itself used an inference for contraction). Instead we interrogate luminal diameter change over time by measuring wall position. The method builds on established MRI techniques that assess motility at single anatomical locations but generates a more comprehensive picture of contractile dynamics. It appears sensitive to even small changes in luminal diameter that conceivably might not activate a pressure sensor on conventional manometric systems(21). Furthermore, it provides excellent spatial resolution at $\sim 1 \mathrm{~mm}$ (or 
the in-plane resolution of the image) potentially enabling us to detect even small localised contractions. This concept has been explored previously in the stomach(24) but here, the combination with image registration makes the approach rapid and feasible in larger time series data. Refinement's like the fitted midline further enable applicability in a wider range of stomach types (e.g. Jshape) and colon which can be convoluted in some individuals.

We applied this technique first in the stomach; the inner and outer stomach wall can be unambiguously identified in these data. Furthermore, from a physiological perspective, contractions migrate along the outer curvature, rather than throughplane, making it an excellent organ to demonstrate proof of concept. In the absence of a gold-standard we used a visual, consensus three-point scoring system to semi-quantitatively grade motility in our datasets. The patients had been prepared with oral mannitol, although the amount of mannitol left in the stomach did vary from subject to subject, helping to provide the desired motility variation in the cohort with which to test our technique. This was intentional as a uniform mannitol preparation would have limited the range of motility encountered and introduced spectrum bias into the investigation of this technique. An additional source of variability was the underlying diagnosis of Crohn's which we have previously found to demonstrate varied motility(25). Clearly a larger range of datasets is now required to assess the technique under different physiological situations(26). A limitation of this data was its relatively short duration of only 20s (the length of a breath-hold) and it would have been interesting to review longer ( $>120$ second) scans. However, visual assessment would have become more challenging with the three-point score over such a prolonged time period and thus the validation aspect may have been negatively impacted. We were particularly encouraged that simple visualisation of the plots seemed to agree well with the dynamic data, with visible propagating contractions (figure 2).

We further applied the technique to the colon. The ascending colon is well seen in the angled sagittal plane, and like the stomach, a contraction can be visually seen to propagate with little ambiguity. Based on historical data from Hoad et al. we explored 10 healthy subjects who had undergone MRI with either 1 or $2 \mathrm{~L}$ of Moviprep(15). Moviprep has a prokinetic effect on bowel motility and the variation introduced before and after the preparation allowed for a more effective dynamic range with which to assess the technique(15). The scan duration was 120 s and a previously validated visual semi-structured reporting system was used to provide the ground-truth(19). Good agreement was seen between our summary metrics and visual inspection of the motility maps. Encouragingly, clearly identified contractile events bared a striking resemblance to those seen in conventional manometry maps, although direct comparison against manometry is an important next step. Reassuringly, the identification of larger contractile events on either of the plots could be confirmed by visual inspection of the original data (Figure 4E\&F).

A number of considerations remain as the technique is developed. The best method to summarise the motility data is important. In this study, we simply calculated an aggregate value under/over a threshold with the NCP/CVD metrics respectively to capture the presence of motility in the colon. However, this is 
"blind" to important data such as the relationship between the contractions, which could better be teased out by alternative methods of interpreting the plot data. It is important to recognise also that the derivation of the motility maps itself introduces complexity. We propose two methods here based on 1) normalised diameter change alone and 2) combined velocity and deformation. Although the results from these two methods were highly correlated, it may be that each has particular advantages in different clinical situations. Both solutions are pragmatic and appear robust to variations in bowel calibre or length but additional investigation is needed to optimise visualisation and data summation. The concept of bowel wall expansion or relaxation was not explored here but the concept of tone is interesting and may be inferred in these data but this falls outside the scope of this investigation and points to future steps in exploring this technique.

The use of empirical thresholds to account for artefacts like noise are sub-optimal but often used. A threshold of $10 \mathrm{~mm} \mathrm{Hg}$ for example has been used in manometry studies(27) to identify significant contractions and, where used, it is essential to report settings in the published work so that they might be repeated by others. In practice, initial visualisation of the plot may be a key driver in how we move forward with this technique and in this regard, would align with the substantial body of expertise drawn from manometry. The total number of contractions per minute might be calculated however we found this challenging in many datasets where a clear contraction could not be resolved. Large events in the colon like high amplitude propagating contractions are also unlikely to be seen in such a short time series. Like high-resolution manometry, this technique's outputs lend themselves well to automated analyses using emerging techniques like machine learning in the future.

The obvious comparator to validate the technique would be manometry. However it must be remembered that a measure of pressure is not necessarily equivalent to a change in bowel diameter and therefore concurrent measures with dedicated MR compatible equipment would be needed to examine agreement between the techniques. In parallel, well controlled clinical studies examining the predictive ability of such MR based techniques will also be valuable for assessing efficacy in practice. Correspondence with the status quo is not necessarily required to demonstrate clinical utility.

For this initial proof of concept study, we did not include the small bowel. Following a specific small bowel loop for more than a few centimetres is challenging with MR due to its convoluted nature and the large amount of throughplane motion produced. As scanning technology improves we will likely be able to image true $4 \mathrm{D}$ data addressing this issue. However, these technical challenges remain outside the abilities of current clinical scanners.

In summary, we demonstrate for the first time a systematic approach to measuring spatio-temporal changes in bowel diameter in gastric and colonic datasets. Agreement with experienced readers was high and the technique may help address the need for objective and systematic measurement of the gut motility. 



\section{Figure text:}

Figure 1. The user is required to place a midline $(M)$ and delineate the inner $\left(L_{1}\right)$ and outer ( $L_{2}$ ) wall of the GI tract to encompass the lumen (as per figure A). The STMM will automatically generate a series of nodes ( $N$ ) at a user determined interval shown here as red dots (B). A series of lines, perpendicular to the midline (M) are generated at each node point to intersect with the lumen. The diameter and position of each line is recorded at each time point. Deformation fields are used to propagate $M, L 1 \& L 2$ to the next time point where the process is repeated without user intervention. The user can set the node spacing to a range of values with 10, 5 and 1 pixel spacing's demonstrated in figures $D-F$.

Figure 2. An example of a contractile stomach (A) the corresponding Normalised Contraction plot (NCP) (B) and Combined Velocity Deformation (CVD) (C), all showing regions of activity in the distal stomach for the duration of the study. 'P' and ' $D$ ' represent proximal and distal ends of the stomach respectively. A hypo-motile stomach is shown in (D). Again, the corresponding NCP is presented (E) along with the CVD (F). Here markedly less contractile activity is seen. Both subjects had a diagnosis of Crohn's but the cause of the apparent dysmotility is unknown. In all plots, the summary metric to assess motility is presented.

Figure 3. Correlation between combined gastric motility visual score NCP (A) and CVD (B).

Figure 4. An example of a contractile ascending colon $(A)$ the corresponding NCP (B) and $C V D(C)$. ' $P$ ' and ' $D$ ' represent proximal and distal ends of the colon respectively. This case attracted high motility scores and the plot shows bursts of contractile activity throughout the time series, especially in the final 40 s of the scan. An example of a colon with a more discrete contraction is shown in (D). The corresponding NCP is presented (E) along with the CVD (F). Here the colon is relatively quiescent for the first 100s of the scan with a large contractile event at 105s. For all plots the summary motility metric is presented. Overall CVD presents more regions of motility primarily because it can look at expansion as well as contraction.

Figure 5. Correlation between colonic visual motility score and NCP (A) and CVD $\operatorname{map}(B)$. 


\section{Appendix 1}

\begin{tabular}{|l|l|l|l|}
\hline Use & Stomach & Stomach & Colon \\
\hline Manufacturer & Siemens 1.5T & Philips 3T & Philips 1.5T \\
\hline Sequence name & TrueFISP & BTFE & BTFE \\
\hline Plane & Coronal & Coronal & Sagittal \\
\hline Field of view $(\mathrm{mm})$ & Variable & Variable & $330 \times 228 \mathrm{~mm}^{2}$ \\
\hline No. Slices & 20 & 20 & 120 \\
\hline Stacks & $6-16$ & $6-15$ & 1 \\
\hline Repetition time $(\mathrm{ms})$ & 3.85 & 1.96 & 3.0 \\
\hline Echo time (ms) & 1.93 & 0.98 & 1.5 \\
\hline $\begin{array}{l}\text { In plane pixel } \\
\text { resolution }\end{array}$ & $2.1 \times 1.6$ & $2 \times 2.4$ & $1.5 \times 1.5$ \\
\hline Slice thickness $(\mathrm{mm})$ & 10 & 10 & 15 \\
\hline Slice gap $(\mathrm{mm})$ & 10 & 10 & $\mathrm{NA}$ \\
\hline Averages & 1 & 1 & 1 \\
\hline Flip angle & 61 & 45 & 70 \\
\hline
\end{tabular}

\section{References}

1. Grønlund D, Poulsen JL, Sandberg TH, et al. Established and emerging methods for assessment of small and large intestinal motility. Neurogastroenterol Motil. 2017;29(7)

2. Menys A, Atkinson D, Odille F, et al. Quantified terminal ileal motility during MR enterography as a potential biomarker of Crohn's disease activity: a preliminary study. Eur Radiol. 2012;22(11):2494-2501

3. Bickelhaupt S, Froehlich JM, Cattin R, et al. Differentiation between active and chronic Crohn's disease using MRI small-bowel motility examinations Initial experience. Clin Radiol. 2013;68(12):1247-1253

4. Hahnemann ML, Nensa F, Kinner S, et al. Improved Detection of Inflammatory Bowel Disease by Additional Automated Motility Analysis in Magnetic Resonance Imaging. Invest Radiol. 2015;50(2):67-72

5. Unger MM, Hattemer K, Möller JC, et al. Real-time visualization of altered gastric motility by magnetic resonance imaging in patients with Parkinson's disease. Mov Disord. 2010;25(5):623-628

6. Ohkubo H, Kessoku T, Fuyuki A, et al. Assessment of small bowel motility in patients with chronic intestinal pseudo-obstruction using cine-MRI. Am J Gastroenterol. American College of Gastroenterology; 2013;108(7):1130-1139 
7. Lam C, Chaddock G, Marciani L, et al. Colonic response to laxative ingestion as assessed by MRI differs in constipated irritable bowel syndrome compared to functional constipation. Neurogastroenterol Motil. 2016;28(6):861-870

8. Odille F, Menys A, Ahmed A, Punwani S, Taylor SA, Atkinson D. Quantitative assessment of small bowel motility by nonrigid registration of dynamic MR images. Magn Reson Med. 2012;68(3):783-793

9. Bickelhaupt S, Cattin R, Froehlich JM, et al. Automatic detection of small bowel contraction frequencies in motility plots using lomb-scargle periodogram and sinus-fitting method-initial experience. Magn Reson Med. 2014; 71(2):628-34 10. Hahnemann ML, Nensa F, Kinner S, Gerken G, Lauenstein TC. Motility mapping as evaluation tool for bowel motility: Initial results on the development of an automated color-coding algorithm in cine MRI. J Magn Reson Imaging. 2014; Feb;41(2):354-60

11. Cullmann JL, Bickelhaupt S, Froehlich JM, et al. MR imaging in Crohn's disease: correlation of MR motility measurement with histopathology in the terminal ileum. Neurogastroenterol Motil. 2013;25(9):749-e577

12. Plumb AA, Menys A, Russo E, et al. Magnetic resonance imaging-quantified small bowel motility is a sensitive marker of response to medical therapy in Crohn's disease. Aliment Pharmacol Ther. 2015;42(3):343-355

13. Dinning PG. A new understanding of the physiology and pathophysiology of colonic motility? Neurogastroenterol Motil. 2018 Nov;30(11):e13395

14. Dinning PG, Wiklendt L, Maslen L, et al. Colonic motor abnormalities in slow transit constipation defined by high resolution, fibre-optic manometry. Neurogastroenterol Motil. 2015;27(3):379-388

15. Hoad CL, Menys A, Garsed K, et al. Colon wall motility: comparison of novel quantitative semi-automatic measurements using cine MRI. Neurogastroenterol Motil. 2015; 2016 Mar;28(3):327-35

16. Menys A, Taylor SA, Emmanuel A, et al. Global Small Bowel Motility: Assessment with Dynamic MR Imaging. Radiological Society of North America; 2013;269(2):443-450

17. Menys A, Hamy V, Makanyanga J, et al. Dual registration of abdominal motion for motility assessment in free-breathing data sets acquired using dynamic MRI. Phys Med Biol. 2014;59(16):4603-4619.

18. Bickelhaupt S, Froehlich JM, Cattin R, et al. Software-supported evaluation of gastric motility in MRI: A feasibility study. J Med Imaging Radiat Oncol. 2014;58(1):11-17

19. Marciani L, Garsed KC, Hoad CL, et al. Stimulation of colonic motility by oral PEG electrolyte bowel preparation assessed by MRI: comparison of split vs single dose. Neurogastroenterol Motil. Wiley-Blackwell; 2014;26(10):1426-1436.

20. Fox MR, Kahrilas PJ, Roman S, et al. Clinical measurement of gastrointestinal motility and function: who, when and which test? Nat Rev Gastroenterol Hepatol. Nature Publishing Group; 2018; 2018 Sep;15(9):568-579

21. Dinning PG. A new understanding of the physiology and pathophysiology of colonic motility? Neurogastroenterol Motil. Wiley/Blackwell (10.1111); 2018;e13395

22. Hahnemann ML, Nensa F, Kinner S, et al. Quantitative assessment of small bowel motility in patients with Crohn's disease using dynamic MRI. Neurogastroenterol Motil. 2015;27(6):841-848 
23. Menys A, Keszthelyi D, Fitzke H, et al. A magnetic resonance imaging study of gastric motor function in patients with dyspepsia associated with Ehlers-Danlos Syndrome-Hypermobility Type: A feasibility study. Neurogastroenterol Motil. 2017;29(9):e13090

24. Bharucha AE, Manduca A, Lake DS, et al. Gastric motor disturbances in patients with idiopathic rapid gastric emptying. Neurogastroenterol Motil. 2011;23(7):617-e252

25. Menys A, Makanyanga J, Plumb A, et al. Aberrant Motility in Unaffected Small Bowel is Linked to Inflammatory Burden and Patient Symptoms in Crohn's Disease. Inflamm Bowel Dis. 2016;22(2):424-432

26. Dinning PG, Zarate N, Szczesniak MM, et al. Bowel preparation affects the amplitude and spatiotemporal organization of colonic propagating sequences. Neurogastroenterol Motil. 2010;22(6):633-e176

27. Bortolotti M, Annese V, Coccia G. Twenty-four hour ambulatory antroduodenal manometry in normal subjects (co-operative study). Neurogastroenterol Motil. 2000;12(3):231-238 
Figure 1
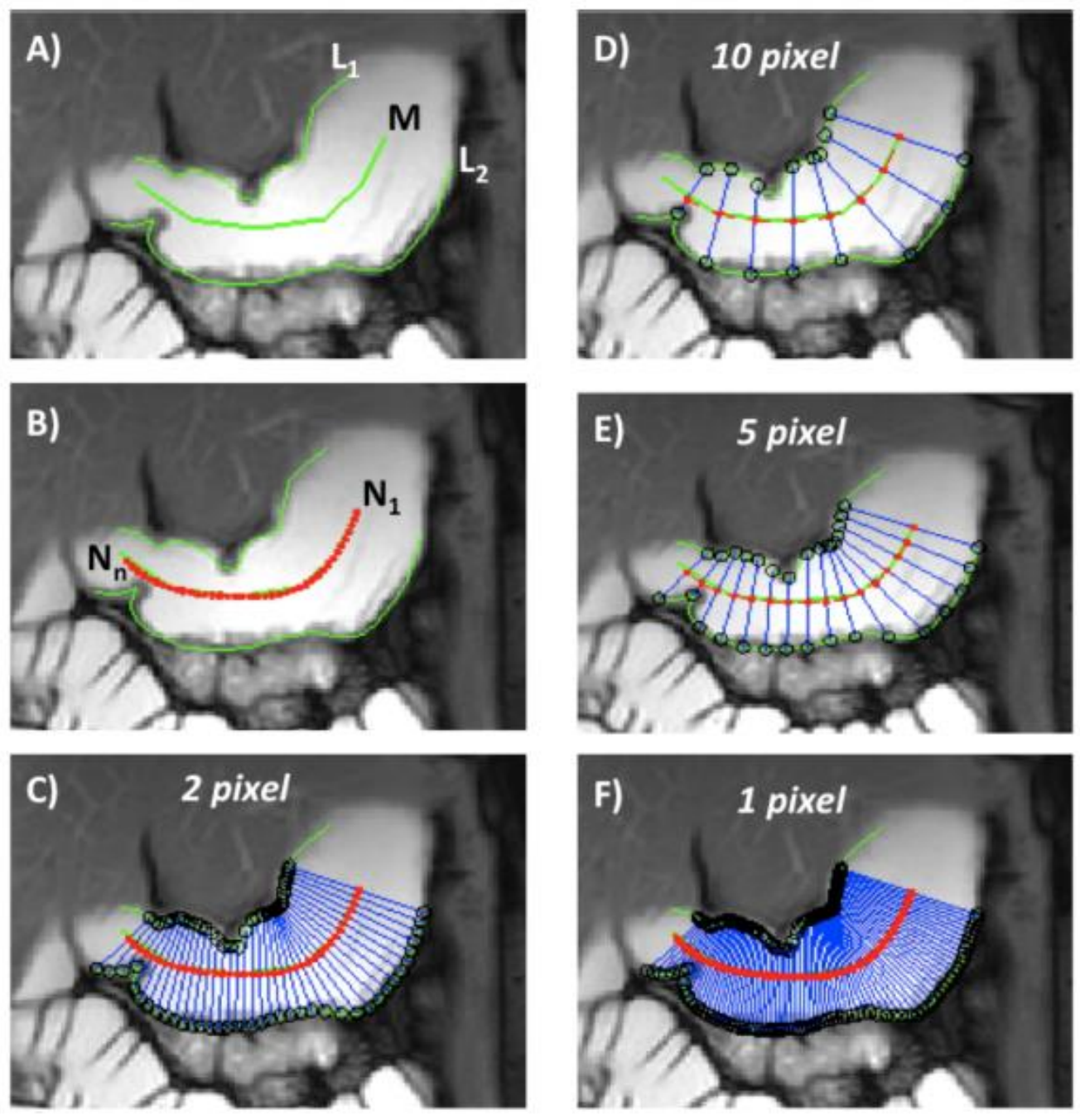
Figure 2
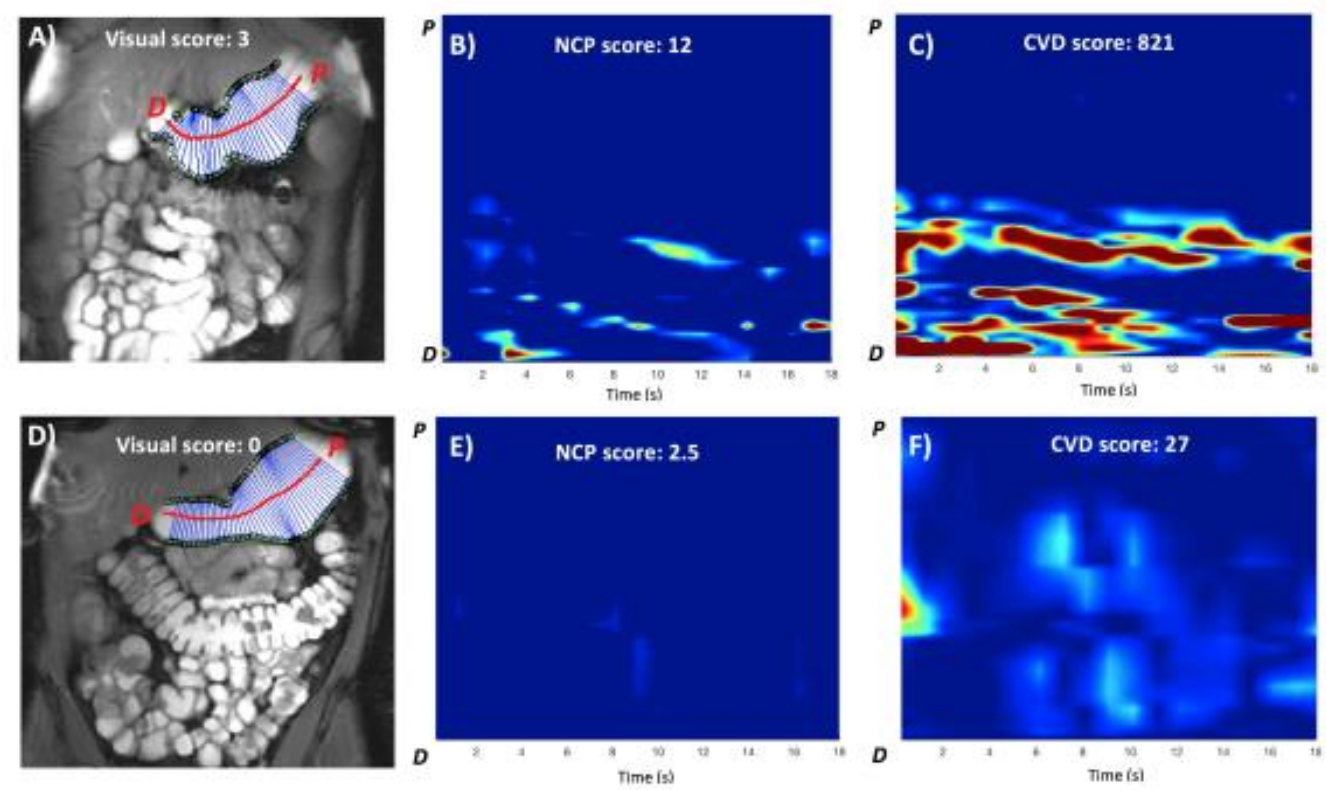
Figure 3

A)

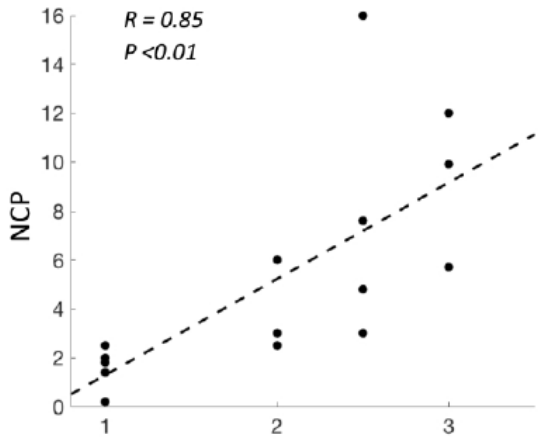

Visual Motility score
B)

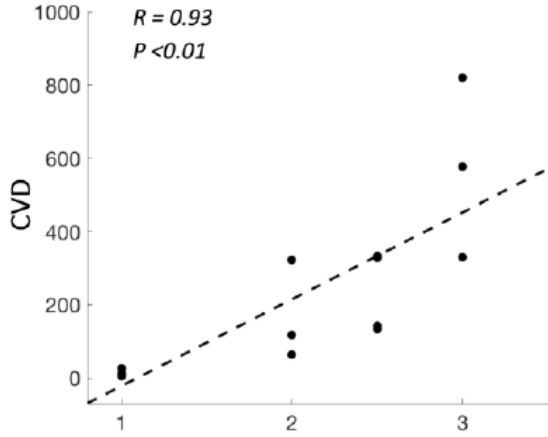

Visual Motility score 
Figure 4
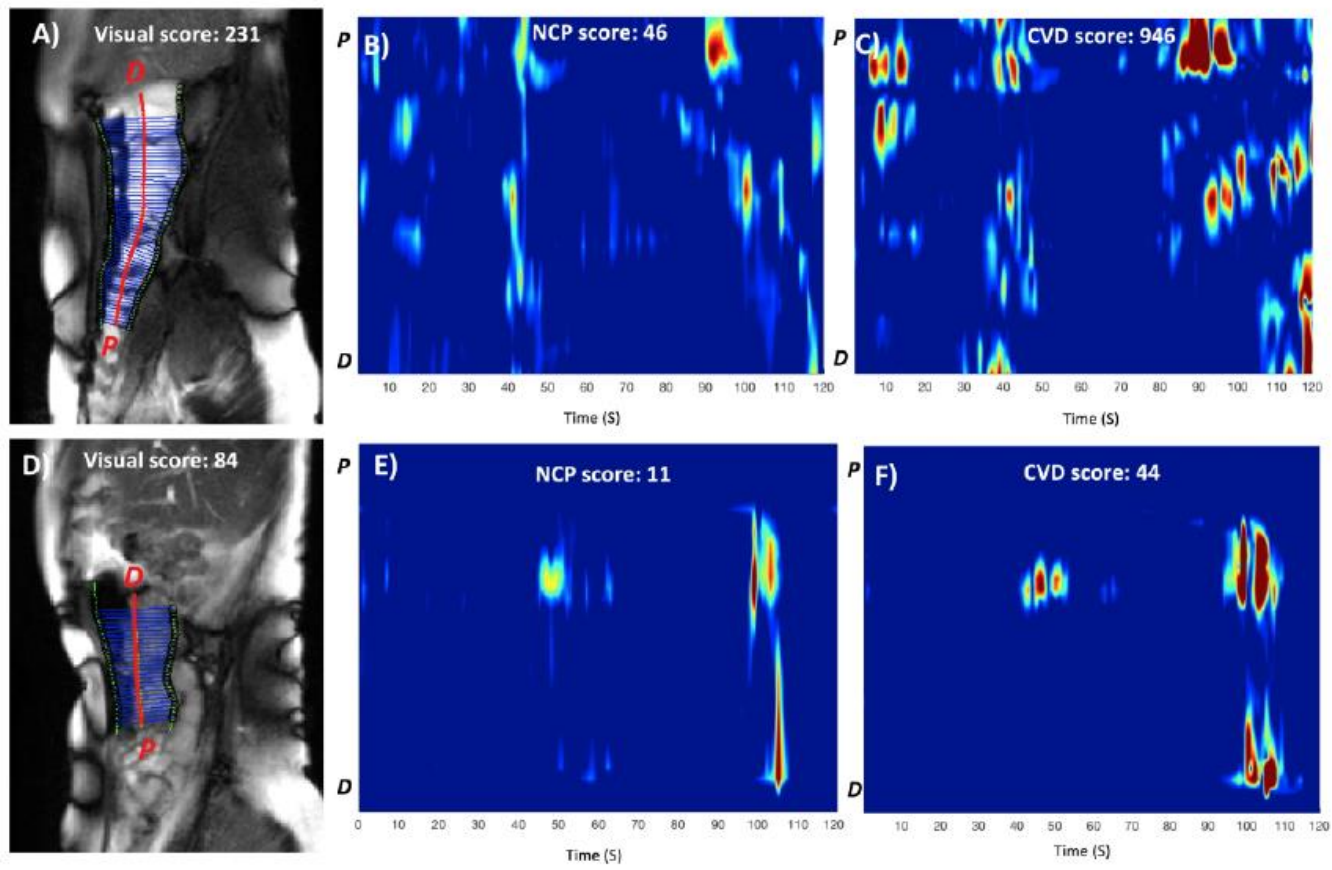
Figure 5

A)

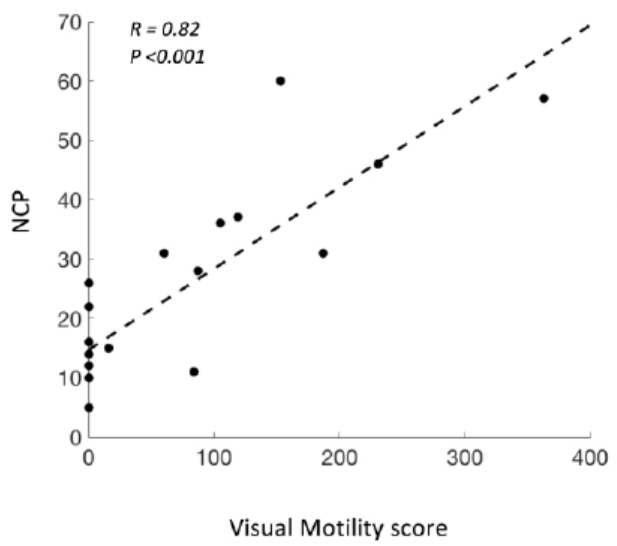

B)

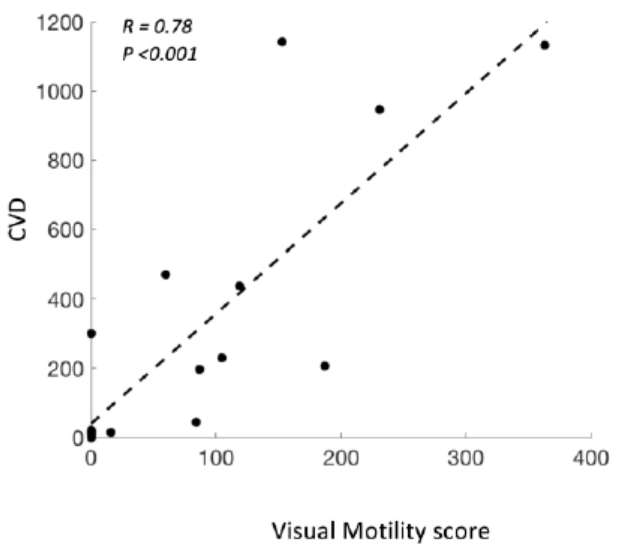

